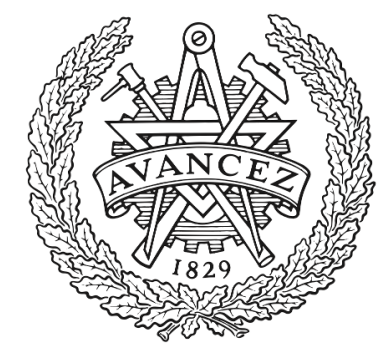

\title{
CHALMERS
}

UNIVERSITY OF TECHNOLOGY

\section{Bit error probability of coherent M-ary PSK over flat Rayleigh fading channels}

Downloaded from: https://research.chalmers.se, 2023-04-26 13:01 UTC

Citation for the original published paper (version of record):

Lassing, J., Ström, E., Agrell, E. et al (2005). Bit error probability of coherent M-ary PSK over flat

Rayleigh fading channels. IEE Electronic Letters, 41(21): 1186-1187. http://dx.doi.org/10.1049/el

N.B. When citing this work, cite the original published paper. 


\section{Bit error probability of coherent $M$-ary PSK over flat Rayleigh fading channels}

Johan Lassing, Erik G. Ström, Erik Agrell, and Tony Ottosson

J. Lassing is with Qamcom Technology, Gruvgatan 8, SE-421 30 Västra Frölunda, Sweden (e-mail: johan.lassing@qamcom.se). E. G. Ström, E. Agrell and T. Ottosson are with the Communication Systems group at Chalmers University of Technology in Gothenburg, Sweden. 


\begin{abstract}
Systems using coherent multilevel PSK to communicate over flat Rayleigh fading channels are considered. We present a closed-form expression for the exact bit error probability when the binary reflected Gray code is used to map bits to symbols. The result is a general, simple, and correct expression, which has not been previously available in the literature. A comparison between this new expression and previous approximate expressions shows that the difference is more significant for fading channels than for Gaussian channels.
\end{abstract}

\title{
I. INTRODUCTION
}

This paper deals with communication systems that uses $M$-ary phase-shift keying ( $M$-PSK) to transmit information over flat Rayleigh fading communication channels. Communication systems employing $M$-PSK have been thoroughly studied in the literature for many different types of channels, see, e.g., [2-4]. In a recent publication [1], a method to evaluate the exact bit error probability (BEP) of $M$-PSK systems was presented for additive, white Gaussian noise (AWGN) channels. Even though the correct expression differs from previously published results on the topic, the difference is only noticeable at very low signal-to-noise ratios (SNR). However, for Rayleigh fading channels the instantaneous SNR is occasionally very small and the discrepancy between the previous and the new expressions may become significant. It is the aim of this paper to establish in what way the new results will affect the exact BEP of $M$-PSK over flat Rayleigh fading channels. In particular, we address the problem of evaluating the bit error probability (BEP) of a coherent $M$-PSK system and present a closed-form expression for the exact BEP of $M$-PSK for any $M=2^{m}$, where $m$ is a positive integer.

\section{System Model AND Symbol ERror Probability}

During a signalling interval the $M$-PSK transmitter can output one out of $M$ signals with quadrature modulation of the form

$$
s_{k}=\sqrt{m E_{b}} e^{j \frac{2 \pi}{M} k}, \quad k=0,1, \ldots M-1,
$$

where $E_{b}$ is the energy per transmitted bit. In the flat Rayleigh channel model, the channel is assumed to introduce two random variables in the signal observed by the receiver; a random fluctuation of the received signal energy and phase and an additive, white Gaussian noise 
component, as seen in the system block diagram of Figure 2. Assuming that the receiver is able to perfectly track the phase of the channel, the detector in the receiver observes the signal $r=|a| s+n$, where $a$ is the complex channel coefficient. The random variable $|a|$ has a Rayleigh probability density function with mean $\sqrt{\pi / 2}$ and $n=n_{I}+j n_{Q}$ is a complex Gaussian noise variable with $n_{I}$ and $n_{Q}$ being identically distributed zero-mean Gaussian random variables with variance $N_{0} / 2$, where $N_{0} / 2$ is the double-sided noise power spectral density.

Each signal alternative is associated with a decision region $S_{k}$, and the receiver determines what $S_{k}$ the received signal $r$ falls within and outputs as its estimate $\hat{s}$ the corresponding signal $s_{k}$. A symbol error is said to occur if the estimate $\hat{s}$ differs from the transmitted signal $s$. We make the reasonable assumption that all signals are equally likely to be selected for transmission, i.e., $\operatorname{Pr}\left\{s=s_{k}\right\}=1 / M$ and we also assume that the receiver uses a maximum likelihood (ML) symbol detector.

\section{Bit ERror Probability for FAding Channels}

When the average probability of bit errors is considered, the mapping of bits onto symbols becomes important. In general, for a system using $M$-PSK with coherent ML detection, the average bit error probability is given by [1]

$$
P_{\mathrm{b}}=\frac{1}{m} \sum_{k=1}^{M-1} \bar{d}(k) P(k)
$$

where the function $\bar{d}(k)$ depends only on the bit-to-symbol mapping and the function $P(k)$ captures the influence of the channel on the error probability. Note that (2) is valid for any (memoryless) channel and to calculate $P_{\mathrm{b}}$, we need to find valid and tractable expressions for $\bar{d}(k)$ and $P(k)$.

The probability $P(k)$ is the probability that the detected signal is found within the wedge in the signal space delimited by angles $(2 k-1) \pi / M$ and $(2 k+1) \pi / M$ (assuming that $s_{0}$ was transmitted). By defining $\theta_{k}^{-} \triangleq(2 k-1) \pi / M$ and $\theta_{k}^{+} \triangleq(2 k+1) \pi / M$ we can find $P(k)$ directly 
using the method described in [2, pp. 223],

$$
P(k)= \begin{cases}1-I\left(\frac{\pi}{M}\right), & k=0, \\ \frac{1}{2}\left(I\left(\theta_{k}^{-}\right)-I\left(\theta_{k}^{+}\right)\right), & k=1,2, \ldots, \frac{M}{2}-1, \\ I\left(\frac{(M-1) \pi}{M}\right), & k=\frac{M}{2}\end{cases}
$$

and $P(k)=P(M-k)$ for $k=M / 2+1, \ldots, M-1$. The function $I(\theta)$ is given, for the interval of primary interest $0<\theta<\pi$, as

$$
I(\theta)=1-\frac{\theta}{\pi}-\beta(\theta)\left(\frac{1}{2}+\frac{1}{\pi} \arctan (\beta(\theta) \cot \theta)\right)
$$

after defining

$$
\beta(\theta) \triangleq \sqrt{\frac{m \bar{\gamma} \sin ^{2} \theta}{1+m \bar{\gamma} \sin ^{2} \theta}},
$$

where $\bar{\gamma}=E_{b} / N_{0}$ is the average energy-to-noise ratio per bit.

The function $\bar{d}(k)$ is called the average distance spectrum (ADS) and is defined as the average number of bit positions differing between signal alternatives separated by $k$ steps along the $M$ PSK circle. In order to evaluate the BEP of an $M$-PSK system, we must specify the particular bit-to-symbol mapping used, since the choice of mapping will affect the BEP. A commonly encountered way of mapping bits to symbols is by means of the binary reflected Gray code (BRGC). In [1, eq. (7)] a closed-form for the ADS of the BRGC is given for all integers $k$,

$$
\bar{d}(k)=2\left|\frac{k}{M}-\left\lfloor\frac{k}{M}\right\rceil\right|+2 \sum_{i=2}^{m}\left|\frac{k}{2^{i}}-\left\lfloor\frac{k}{2^{i}}\right\rceil\right|
$$

where $\lfloor x\rceil$ rounds $x$ to the nearest integer (ties are resolved arbitrarily).

By combining (6) with (2) and the symmetric relation $P(k)=P(M-k)$, we obtain

$$
P_{\mathrm{b}}=\frac{1}{m} \sum_{k=1}^{M-1}\left(2\left|\frac{k}{M}-\left\lfloor\frac{k}{M}\right\rceil\right|+2 \sum_{i=2}^{m}\left|\frac{k}{2^{i}}-\left\lfloor\frac{k}{2^{i}}\right\rceil\right|\right) P(k) .
$$

This equation, together with (3) and (4), provides a closed-form expression for the exact BEP of coherent $M$-PSK over flat Rayleigh fading channels. 


\section{COMMENTS AND CONCLUSIONS}

A commonly encountered approach in the literature when evaluating the BEP of coherent $M$-PSK systems, see e.g., [3, sec. IV], is to use the Hamming weights of the binary labels assigned to the symbols, rather than the ADS. As was shown in [1], this method only generates approximate results. However, for the Gaussian case, the difference is very small and almost not noticeable. In Figure 1, the ratio between the BEP evaluated using the Hamming weights, $w_{H}(k)$, and the BEP evaluated using the ADS, $\bar{d}(k)$, is shown for the Gaussian channel and the flat Rayleigh fading channel. We note that the approximation of using $w_{H}(k)$ instead of $\bar{d}(k)$ is still accurate, although much less accurate than for the AWGN channel. However, since there is a closed-form available for the ADS, there is little motivation for the approximation of using $w_{H}(k)$. From Figure 1 we also observe that the difference between the approximation and the exact BEP for the fading channel is noticeable over the whole range of practical values of the bit energy-to-noise ratio, which is not the case for the AWGN channel.

\section{REFERENCES}

[1] J. Lassing, E. G. Ström, E. Agrell, and T. Ottosson, "Computation of the exact bit error rate of coherent $M$-ary PSK with Gray code bit mapping," IEEE Transactions on Communications, vol. 51, no. 11, pp. 1758-1760, Nov. 2003.

[2] M. K. Simon and M. S. Alouini, Digital Communications over Fading Channels-A Unified Approach to Performance Analysis, John Wiley \& Sons, 2000.

[3] J. Sun and I. S. Reed, "Linear diversity analyses for M-PSK in Rician fading channels," IEEE Transactions on Communications, vol. COM-51, no. 11, pp. 1749-1753, Nov. 2003.

[4] P. J. Lee, "Computation of the bit error rate of coherent $M$-ary PSK with Gray code bit mapping," IEEE Transactions on Communications, vol. COM-34, no. 5, pp. 488-491, May 1986. 


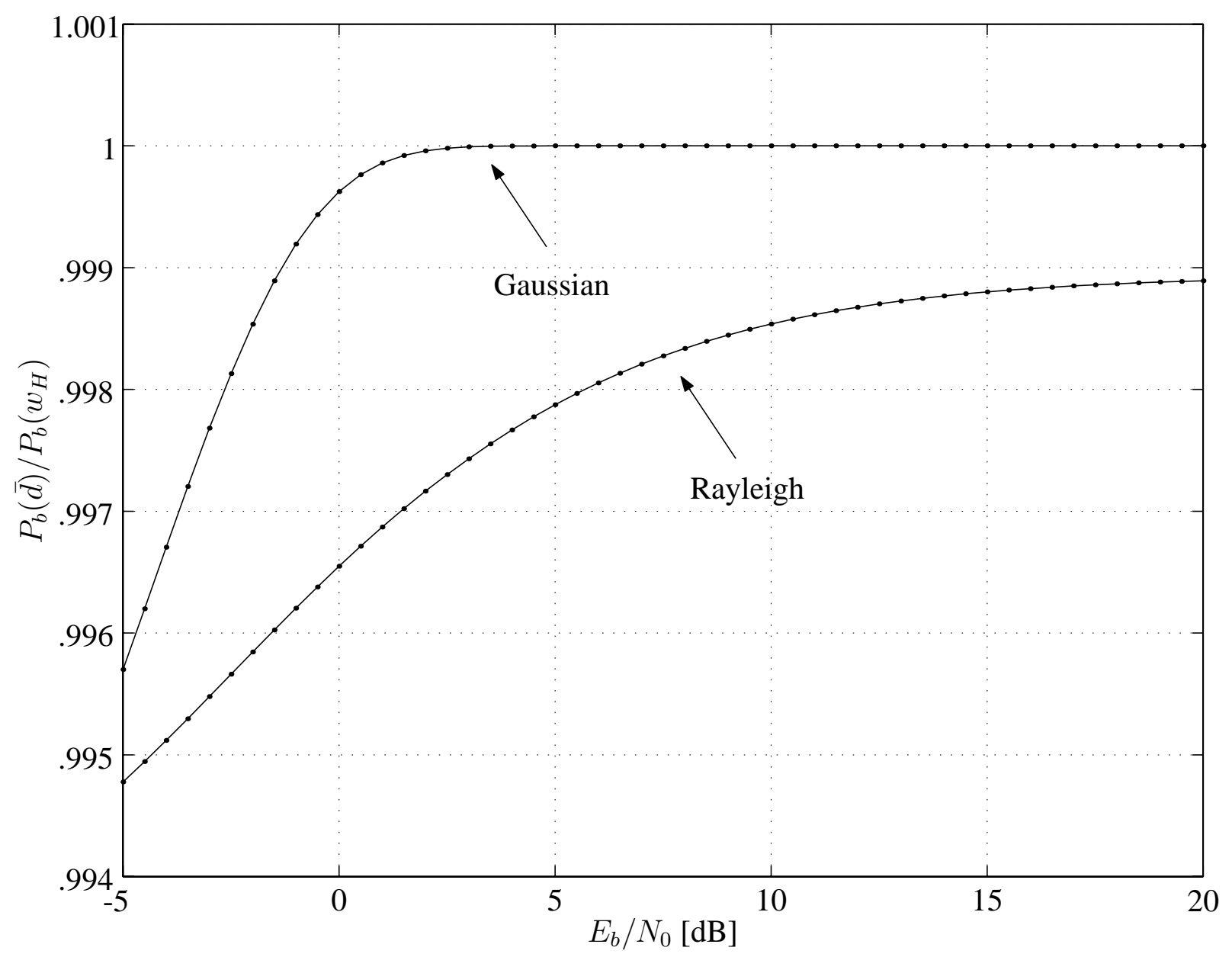

Fig. 1. The graph indicates the ratio $P_{b}(\bar{d}) / P_{b}\left(w_{H}\right)$ for $M=16$, where $P_{b}\left(w_{H}\right)$ is (2)-(5) evaluated using the Hamming weights of the BRGC labels in place of the ADS, and $P_{b}(\bar{d})$ is (7) with (3)-(5).

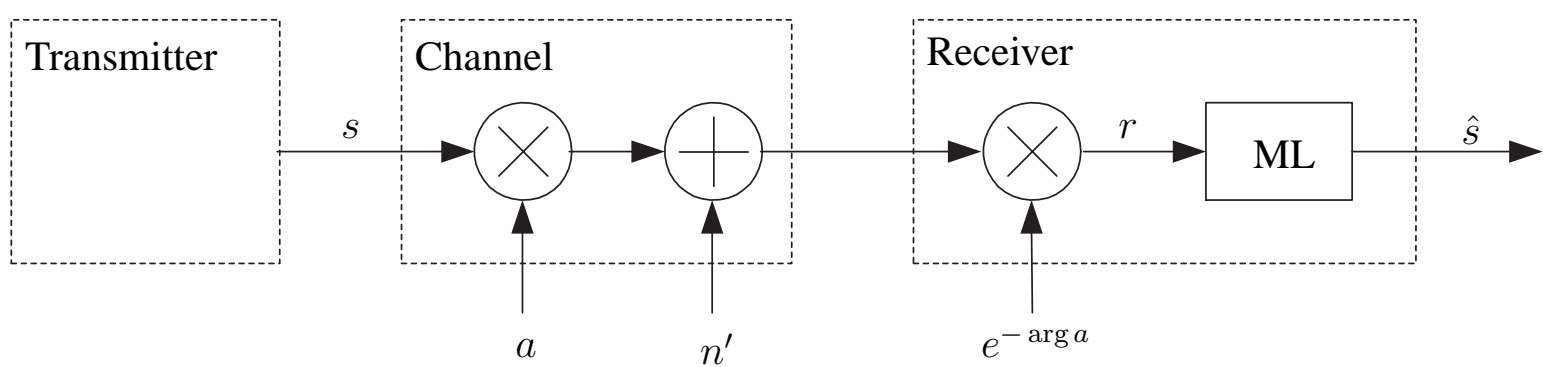

Fig. 2. Schematic block diagram of a communication system communicating over a Rayleigh fading channel. 